\title{
FATORES ASSOCIADOS A OCORRÊNCIA DE INFILTRAÇÕES E EXTRAVASAMENTOS INTRAVENOSAS EM CRIANÇAS COM DISPOSITIVOS INTRAVASCULARES PERIFÉRICOS EM UMA UNIDADE DE CLÍNICA CIRÚRGICA
}

\author{
$\underline{\text { Manuela de Jesus Souza }}{ }^{1}$; Luciano Marques dos Santos ${ }^{2}$; Sarah Almeida $\operatorname{Santos}^{3}$ e \\ Cleonara Sousa Gomes e Silva ${ }^{4}$ \\ 1. Bolsista PEVIC/UEFS, Graduando do Curso de Enfermagem, Universidade Estadual de Feira de Santana, e-mail: \\ manusouzauefs@gmail.com \\ 2. Orientador, Departamento de Saúde, Universidade Estadual de Feira de Santana, e-mail: lucmarxenfo@ yahoo.com.br \\ 3. Participante do projeto de pesquisa "Segurança do paciente pediátrico e sua família: estudo de tecnologias e eventos \\ adversos relacionados a terapia intravascular periférica. Departamento Saúde, Universidade Estadual de Feira de Santana, e- \\ mail: sarahsantosuefs@gmail.com \\ 4. Participante projeto de pesquisa "Segurança do paciente pediátrico e sua família: estudo de tecnologias e eventos adversos \\ relacionados a terapia intravascular periférica. Departamento Saúde, Universidade Estadual de Feira de Santana, e-mail: \\ cleosilvauefs@gmail.com
}

PALAVRAS-CHAVE: Terapia Intravenosa; Infiltração; Cateterização Intravenosa Periférica

\section{INTRODUÇÃO}

O processo de hospitalização de crianças, por diversas doenças de natureza clínica ou cirúrgica, necessita em muitos casos de assistência terapêutica com a utilização de dispositivos venosos, sendo assim realizado a Cateterização Intravenosa Periférica (CIP). A Terapia Intravenosa (TIV) pode causar eventos adversos, como por exemplo, as complicações intravenosas. A infiltração é uma das complicações mais incidentes entre as crianças podendo estar associada a determinadas características da população pediátrica.

Desta forma, o objetivo desta trabalho foi Descrever a associação entre características da criança, da CIP atual e da TIV utilizada com a ocorrência de infiltrações em crianças com condições cirúrgicas no Hospital Estadual da Criança (HEC) em Feira de Santana.

\section{MATERIAL E MÉTODOS}

Trata-se de um estudo transversal, que foi realizado na unidade de clínica cirúrgica do Hospital Estadual da Criança (HEC) em Feira de Santana na Bahia. Para participar do estudo foram convidadas crianças submetidas à CIP no internamento da unidade de clínica cirúrgica do HEC, no período de abril de 2015 à dezembro de 2016, sendo observado 137 cateteres intravasculares periféricos. Os dados foram coletados por meio de fonte primária através da observação da CIP em cada criança selecionada e secundária através da consulta ao prontuário do paciente, pelos pesquisadores pertencentes ao projeto. Durante a coleta, após a realização da CIP pelos profissionais de enfermagem da unidade em estudo, os dispositivos e a região ao redor do cateter periférico foram observados diariamente, com o objetivo de avaliar as condições da pele, verificando a presença ou a ausência de sinais sugestivos de infiltração.

Os dados coletados no estudo foram tabulados em planilha eletrônica e analisados por meio do programa de estatística, Statistical Package for Social Sciences (SPSS), versão 22.0 e o programa estatístico Open Epi Versão 3.01.

\section{RESULTADOS}


Dentre os 642 dispositivos intravenosos por via periférica para infusão da TIV observadas no campo deste estudo, 137 foram observados na Clínica Cirúrgica. Em relação ao tipo de complicações intravenosa mais frequente foram flebite $(41,9 \%)$ e extravasamento $20,3 \%$.

Em relação ao grau de extravasamento houve uma maior ocorrência do Grau 1 (50\%), seguido do Grau $2(35,7 \%)$ e Grau $3(14,3 \%)$. Quanto ao grau da infiltração, destacou-se a ocorrência do Grau $1(73,3 \%)$, quanto que ao Grau 2 foi de apenas $(26,7 \%)$. Não houve casos de extravasamento Grau 4 nem infiltração Grau 3 e 4 na amostra estudada.

Para a caracterização da amostra foi considerada a primeira ocorrência de flebite em cada criança que apresentou esta complicação, totalizando 34 crianças caracterizadas, neste estudo.

Com relação às características sócio-demográficas das crianças que apresentaram infiltração ou extravasamento, relacionado a idade, houve uma predominância de crianças com idade entre 36 e 83 meses assim como de adolescentes com idade igual ou superior a 132 meses $(28,6 \%)$, a maioria delas eram do sexo masculino 70,6\% (24), 50\% (17) se autodeclararam sendo pardas e foram classificadas de acordo com a condição nutricional como eutróficas $76,5 \%$ (26).

Quanto à condição clínica, a maioria das crianças foi hospitalizada com diagnósticos médicos relacionados ao sistema gastrointestinal (34,5\%), seguidos de afecções do sistema neurológico $(27,6 \%)$ e musculoesquelético $(20,7 \%)$. Em relação ao histórico de prematuridade $94,1 \%$ (32) delas não possuíam história, 79,4\% (27) não apresentavam doenças crônicas, 91,2\% (31) não possuíam edema no membro puncionado, $85,3 \%$ (29) não foram classificadas como hiperativas no momento da punção e $58,8 \%$ (20) tinham até 7 dias como tempo de hospitalização. Sendo que, observou-se que o número mínimo de 1 dia e o máximo de 193, com uma média de 20,79 dias $( \pm 26,1)$.

Nas variáveis relacionadas à TIV prévia, 61,8\% (21) das crianças apresentavam histórico de dificuldade para inserção de CIP, 44,1\% (15) não fizeram o uso de TIV periférica prolongada, $85,3 \%$ (29) utilizaram CIP previamente e 79,4\% (27) já haviam tido complicações relacionadas a TIV anteriormente, sendo que destas: $29,4 \%$ (10) apresentaram antecedente de flebite, 58,8\% (20) de infiltração, 17,6\% (6) de extravasamento, e 14,7\% (5) de obstrução.

Quanto às variáveis relacionadas à PIP atual, 50\% (17) das crianças foram puncionadas no membro superior direito (MSD), em relação ao calibre do cateter 67,6\% (23) foram puncionadas com o de 22 Gauge e através do método direto de punção (91,2\%, n=31). Em relação às condições das veias das crianças estudadas, 61,8\% (21) foram classificadas como visíveis e com trajeto retilíneo, 79,4\% (27) foram classificadas como palpáveis, 64,7\% (22) classificadas fixas no momento da punção e $73,5 \%$ (25) apresentaram veias superficiais. A estabilização do cateter foi realizada em 79,4\% (27) das crianças e utilizaram a cobertura estéril associada ao micropore e nenhuma criança fez uso da tala imobilizadora no membro cateterizado.

Com relação as variáveis relacionadas à TIV utilizada pelas crianças após a CIP, 30,3\% (23) delas fizeram uso de medicamentos irritantes, 44,4\% (22) utilizaram medicamentos vesicantes e $40 \%$ (22) não utilizaram medicamentos nem irritantes nem vesicantes. Em relação as soluções utilizadas, $60 \%$ (25) utilizaram soluções de natureza vesicante, e 39,7\% (23) fizeram uso de soluções que não foram classificadas nem como irritantes nem como vesicantes. Sobre a quantidade de medicações utilizadas, observou-se que 35,4\% (17) utilizaram até dois medicamentos irritantes, 44,\% (12) utilizaram até dois vesicantes e 23,5\% (12) utilizaram até dois dos que não foram classificados nem como irritantes nem como vesicantes. Já em relação a utilização de soluções a maioria também utilizou até duas soluções: 64,3\% (9) soluções vesicantes e $39,7 \%$ (23) soluções que não eram nem irritantes nem vesicantes. A maioria das crianças 35,5\% (19) tiveram o método de administração misto (Bomba de infusão+bureta+bolus). $\quad 42,1 \%$ (16) dessa infusão da TIV ocorreu de forma 
contínua+intermitente, e $34,1 \%$ (29) permaneceram com o CIP por intervalo menor que 72 horas.

Para o cruzamento das variáveis foram consideradas todas as punções estudadas, ou seja, àquelas em que ocorreram infiltração/extravasamento $(\mathrm{N}=34)$ e às que não apresentaram nenhuma complicação $(\mathrm{N}=75)$.

Com relação às características relacionadas à criança, observou-se significância estatistica ( $\mathrm{p}=0,001)$ em 20,8\% (10) das crianças que se auto declararam de cor branca ou vermelha que apresentaram esta complicação e também para aquelas crianças do sexo masculino $(\mathrm{p}=0,038) 39,3 \%(24)$.

Não foram encontradas diferenças estatísticas com relação às variáveis: idade, sexo, condição nutricional, história de prematuridade, presença de edema, hiperatividade da criança e tempo de hospitalização. Porém, esta complicação ocorreu com maior frequência nas punções realizadas em crianças menores de 7 anos $(39,5 \%)$, do sexo masculino (35,8\%), desnutridas $(26,7 \%)$ sem histórico de prematuridade $(31,7 \%)$, sem presença de edema $(37,5 \%)$ ou doença crônica $(46,7 \%)$, que não estavam hiperativas durante a punção $(34,9 \%)$ e estavam internadas por um tempo inferior a 8 dias.

No que se refere às características da TIV prévia, observou-se diferenças estatísticas entre os grupos para histórico de dificuldade para inserção de CIP $(p=0,009)$ e com antecedentes de complicação $(\mathrm{p}=0,037)$. Em relação ao tipo de cateter prévio houve uma significância de p>0,999. Não foram observadas significâncias estatísticas para uso de TIV prévia, TIV prolongada e tipos de complicações prévias.

Entretanto, ocorreram mais infiltrações/extravasamento nas punções realizadas em crianças que fizeram uso de TIV prévia (30,2\%), utilizaram CIP previamente (30,8\%), tiveram antecedentes de complicações por infiltração $(37,7 \%)$.

As variáveis relacionadas à PIP atual utilizada não influenciaram significativamente no desenvolvimento de flebite. No entanto, esta complicação ocorreu com mais frequência nas punções realizadas nos membros superiores (MMSS) $(31,3 \%)$, com rede venosa visível (28,8\%), palpável $(31,4 \%)$, retilínea $(28,4 \%)$, fixa $(33,4 \%)$ e superficial $(34,7 \%)$; nas punções em que utilizou-se cateteres de $22 \mathrm{G}(42,9 \%)$, método direto $(34,1 \%)$ e com estabilização do cateter $(38,9 \%)$.

Em relação as variáveis relacionadas a TIV atual, houve significância estatística entre o grupo de crianças que fez uso de medicamentos não irritantes/vesicantes $(p=0,045)$, utilizaram solução vesicante $(\mathrm{p}=0,015)$ e também solução não irritante/vesicante $(\mathrm{p}=0,042)$.

Mas houve uma prevalência de complicações nas as punções realizadas em crianças que fizeram uso de medicamentos irritantes $(33,3 \%)$, e que utilizaram até dois em medicamentos em todas as classes medicamentosas.

\section{DISCUSSÃO}

De acordo com o estado da arte elaborado houve dificuldade para encontrar estudos nacionais e internacionais que abordam os fatores associados à ocorrência de infiltrações em crianças e adolescentes e estes também ainda não estão muito claros.

Este evento adverso pode estar associado às características sociodemográficas da criança e do adolescente, histórico da TIV, características da cateterização e da TIV em utilizada.

Com relação as características da criança e adolescente, a literatura cita a faixa etária(TRIPATHI; KAUSHIK; SINGH, 2008; PARK et al., 2016; ABUSAFIA; BOZTEPE, 2017) e sexo(DANSKI et al., 2016b). Um estudo quase-experimental realizada na Coreia (PARK et al., 2016) observou diferenças estatísticas entre idade e ocorrência de infiltração. Este evento adverso foi maior em crianças com idade inferior a 1 ano $(p=0,007)$. Neste estudo não foi observada diferença para a variável sexo. Outra coorte prospectiva em neonatos demonstrou que $45,4 \%$ dos cateteres utilizados nos do sexo masculino foram 
retirados devido à infiltração/extravasamento (DANSKI et al., 2016b) o que pode ser observado no presente estudo foi uma maior prevalência desta complicação em crianças do sexo masculino.

Características demográficas não foram estatisticamente significantes para a ocorrência de infiltração em um estudo com crianças e adolescentes paulistas. Neste estudo, a maioria das crianças com infiltração foram de idade escolar, do sexo masculino, eutróficas e com cor de pele parda (JACINTO; AVELAR; PEDREIRA, 2011) e neste presente estudo houve significância estatística para aquelas crianças que se autodeclaram cor branca ou vermelha.

As características demográficas da criança também não foram associadas à ocorrência de infiltração em um estudo realizado na Coreia (PARK; JEONG; JUN, 2016).

No que se refere ao histórico $\boldsymbol{d a} \boldsymbol{T I V}$, autores citam presença de condições predisponentes para o insucesso da CIP, história de complicações decorrentes da TIVe utilização de TIV previamente(JACINTO; AVELAR; PEDREIRA, 2011) e já no presente estudo observou-se diferenças estatísticas entre os grupos para histórico de dificuldade para inserção de CIP $(\mathrm{p}=0,009)$ e com antecedentes de complicação $(\mathrm{p}=0,037)$.

Nas características da cateterização em uso, citam-seo calibre do cateter (ABUSAFIA; BOZTEPE, 2017), reajuste do cateter durante a CIP(JACINTO; AVELAR; PEDREIRA, 2011), local de realização da CIP(MALYON et al., 2014; PARK; JEONG; JUN, 2016; JEONG et al., 2017), tipo de cobertura do cateter (JEONG; PARK; PARK, 2016)e tempo de permanência do cateter in situ(TRIPATHI; KAUSHIK; SINGH, 2008; JACINTO; AVELAR; PEDREIRA, 2011; ABUSAFIA; BOZTEPE, 2017; JEONG et al., 2017).

As variáveis relacionadas à PIP atual utilizada não influenciaram significativamente no desenvolvimento de infiltração. Em relação as variáveis relacionadas a TIV atual, houve significância estatística entre o grupo de crianças que fez uso de medicamentos não irritantes/vesicantes $(\mathrm{p}=0,045)$, utilizaram solução vesicante $(\mathrm{p}=0,015)$ e também solução não irritante/vesicante $(\mathrm{p}=0,042)$.

Houve uma correlação significativa entre o calibre do cateter intravenoso periférico e o tipo de complicação em crianças Turcas $(\mathrm{p}=0,036)$. A infiltração foi maior nos cateteres $22 \mathrm{G}$ (73\%) e 24 G (72,9\%) (ABUSAFIA; BOZTEPE, 2017) assim como observado no presente estudo. Neste estudo, apenas duas CIP foram realizadas em membros inferiores e não apresentaram infiltrações.

\section{CONCLUSÃO}

Considerando o número elevado da ocorrência de infiltração e extravasamento que são complicações da terapia intravenosa encontrado no resultado deste estudo e também nas produções cientificas sobre a temática, torna-se necessário e relevante conhecer os fatores associados à esta ocorrência para que com estudo e produção científica os profissionais, principalmente de Enfermagem, sintam-se seguros para melhoria das suas habilidades e melhores resultados durante sua prática, garantindo a estas crianças e suas famílias uma terapia de qualidade centrada na segurança dos mesmos.

\section{REFERENCIAS}

DIAS, E. F.; et al. Utilização do dispositivo intravenoso periférico intermitente em pediatria. Revista eletrônica de Enfermagem, v. 2, n. 2, 2006. Disponível em: 〈http://www.fen.ufg.br/revista2_2/dipi.html>. Acesso em: 29 nov. de 2015.

GOMES A.V.O.; et al. Punção venosa pediátrica: uma análise crítica a partir da experiência do cuidar em enfermagem. Rev. Enferm Global, v.10, n.3, p. 287-297, jul 2011. Disponível em: 〈http://revistas.um.es/eglobal/article/view/131991/122411>. Acesso em: 03 nov. 2015. 
LIMA, J. A. K.; AVELAR, A. F.; PEDREIRA, M. L. Predisposing factors for infiltration in children submitted to peripheral venous catheterization. Journal of Infusion Nursing. v. 34, n. 6, p. 391-398, 2011.

MACHADO, A. F.; PEDREIRA, M. L. G.; CHAUD, M. N. Eventos adversos relacionados ao uso de cateteres intravenosos periféricos em crianças de acordo com tipos de curativos. Rev. Latino-Am. Enfermagem, v. 16, n. 3, p. 362-367, jun 2008. Disponível em:

$<$ http://www.scielo.br/scielo.php?script=sci_arttext\&pid=S010411692008000300005\&lng=en \&nrm=iso>. Acesso em: 04 nov. 2015.

MARTINS, T. et al. Infusions failures in the use of peripheral venous catheters in children: integrative review. Online Brazilian Journal of Nursing, v. 8, n.1, p. 1953, fev 2009.

Disponível em: <http://www.objnursing.uff.br/index.php/nursing/article/view/j.16764285.2009.1953 >. Acesso em: 09 nov. 2015.

PEREIRA, R. C. C.; ZANETTI, M. L. Complicações decorrentes da terapia intravenosa em pacientes cirúrgicos. Rev. Latino-Am. Enfermagem, v. 8, n. 5, p. 21-27, out 2000.

Disponível em

<http://www.scielo.br/scielo.php?script=sci_arttext\&pid=S010411692000000500004\&lng=pt \&nrm=iso>. Acesso em: 04 nov. 2015. 